ACTA UNIVERSITATIS LODZIENSIS

Folia Litteraria Romanica 16, 2021

https://doi.org/10.18778/1505-9065.16.08

Alma Sokolija

Université de Sarajevo

(iD https://orcid.org/0000-0002-2096-2827

alma.sokolija@ff.unsa.ba

\title{
L'amour et ses expressions dans certains argots du monde
}

\begin{abstract}
RÉSUMÉ
L'objectif de cet article est de montrer un peu comment le langage de l'amour est articulé dans certains argots du monde. L'argot est souvent identifié comme peu tendre dans ses expressions d'amour, surtout celles qui concernent la sexualité. Cependant, la sexualité est perçue différemment suivant les cultures. Ce qui est considéré comme très vulgaire dans une langue et une culture est ressenti comme moins vulgaire dans les autres. Nous aborderons quelques langues culturellement éloignées en essayant de faire des parallèles. En partant de l'hypothèse que nous vivons dans des sociétés majoritairement patriarcales, certaines notions tabous liées à la sexualité restent les mêmes spécialement dans les cultures où le patriarcat est fortement prononcée. Cependant, notre attitude envers ces expressions qui dévoilent une partie intime et souvent inconsciente de nos vies reste non seulement collective (perception du vulgaire, par exemple) mais aussi profondément individuelle.
\end{abstract}

MOTS-CLÉS - les expressions d'amour en argot, les mots tabous exprimant la sexualité en argot, les tabous culturels liés à la sexualité

\section{Love and Its Expressions in Certain Slangs of the World}

\begin{abstract}
SUMMARY
One of the most frequent themes in the world, love, is the subject of many controversies. The aim of this paper is to show a little how the language of love is articulated in certain slangs of the world. Slang is frequently identified as a little crude in its expressions of love which concern mainly sexuality. However, sexuality is differently perceived in different cultures. What is considered to be very vulgar in one language is felt as less vulgar in another language and culture. Based on the assumption that we live in mostly patriarchal societies, some notions of taboos related to sexuality remain the same especially in the cultures where patriarchy is very pronounced. However, our attitude to these expressions - which reveal one intimate and frequently unconscious part of us - remains not only collective (perceiving something as vulgar for example) but also profoundly individual.
\end{abstract}

KEYWORDS - slang love expressions, taboo words expressing sexuality in slangs, cultural taboos related to sexuality 


\section{L'argot ou l'amour à travers la sexualité}

En parlant de l'amour en argot, nous pouvons remarquer que l'amour y est souvent réduit à la sexualité. L'argot ne comporte pas vraiment de mots pour traduire l'attendrissement ( $c f$. Guiraud $1973: 43$ ). Pour ne donner qu'un exemple, Pierre Guiraud, dans son Dictionnaire érotique (Guiraud, 1978a : 15-23), cite 1300 mots et images qu'on peut trouver en argot français pour désigner le 'coït'. Bien sûr, la panoplie y est très large et on y trouve des expressions plus ou moins vulgaires (foutre le con, baiser) mais aussi des euphémismes (le faire, coucher avec) et même des expressions qui ne sont pas vraiment argotiques et qui sont presque poétiques (fêter, goûter les joies de ce monde).

Malgré la richesse du vocabulaire argotique à connotation sexuelles, il est tout à fait commun que ces mots restent tabous et que leur emploi soit mal perçu dans la langue standard même si certains parmi eux sont présents dans les dictionnaires de la langue standard. Ils sont souvent remplacés à tort, chez ceux qui évitent ces expressions, par des euphémismes dont on connaît rarement l'étymologie : fichtre! remplace foutre ! par exemple en tant qu'interjection (Guiraud, 1975 : 112). Et pourtant, il y a une forte disproportion dans la fréquence de l'emploi des vulgarismes et la reconnaissance de leur présence surtout dans la langue de tous les jours. C'est pourquoi nous considérons qu'il est important d'attirer l'attention des étudiants des langues étrangères sur ces expressions argotiques justement pour qu'ils puissent les décoder correctement et éviter les malentendus possibles.

Le côté corporel de l'amour, sa dimension physique, en effet, reste souvent tout simplement occulté et tabouisé. Finalement, l'emploi de l'argot ainsi que des mots argotiques vulgaires sont quelque chose qui touche à la sensibilité personnelle et personne n'est obligé d'utiliser ces mots même s'il les connaît très souvent de façon passive ( $c f$. Sokolija, 2014a : 6). Les vrais argotiers ne rejettent pas ces mots ou plutôt, au contraire, les argots deviennent une sorte de réserve de mots désignant les tabous de la culture générale et de la langue commune, des mots touchant ainsi au sexe, à la folie, à la mort, à la criminalité, à la prostitution et ainsi de suite. Ainsi, nous avons remarqué :

\footnotetext{
Très souvent injustement on associe l'argot au vulgaire et au grossier. C'est le plus souvent un préjugé social qui associe symboliquement les gens qui utilisent les mots argotiques à des gens vulgaires et grossiers. Le mot vulgaire provient d'ailleurs du mot latin vulgus, le 'peuple', le 'bas peuple'. Le latin vulgaire est un latin populaire qui a donné les différentes langues romanes dans les provinces de l'Empire romain. La langue du peuple est la partie la plus dynamique d'une langue qui porte en elle les changements qui vont un jour, tôt ou tard, être pérennisés dans cette langue. Ceci étant dit, les mots argotiques peuvent coexister avec les mots vulgaires et grossiers mais ceci n'est pas nécessairement l'essence d'un argot (Sokolija, 2014a : 9).
}

Quoiqu'il en soit nous restons aujourd'hui confrontés à un paradoxe du monde moderne où l'on trouve d'une part une sexualité omniprésente et explicite dans 
certaines sociétés où, d'autre part, on trouve des cultures où cette même sexualité est extrêmement tabouisée et sacralisée. Ceci rappelle la métaphore du fruit interdit qui devient intéressant parce qu'il implique un conflit d'ordre psychologique/sociologique qui a du mal à trouver sa solution (cf. Sokolija, 2014b : 320). Du moment qu'il y a un tabou ou une interdiction, ils appellent à être transgressés, ce qui mène à une répression de la société attachée à des religions qui maintiennent ces mêmes tabous. Par la suite cela mène à nouveau à des transgressions créant ainsi à un cercle vicieux.

C'est pourquoi la sexualité dans l'argot doit exister d'autant plus dans les pays et les milieux où la sexualité reste un tabou et notre objectif sera de le démontrer, ne serait-ce que très brièvement. Nous essayerons d'en donner des illustrations à travers des mots argotiques liés à l'amour et à la sexualité dans certaines langues. Et ceci n'est pas sans quelques difficultés parce que l'image de l'amour idéalisé et sacralisé, réduit aux sentiments ou strictement à la sphère du mariage, est proportionnelle à un certain manque sur Internet d'exemples argotiques qui concerne la sexualité dans certaines langues. La censure de l'argot sur Internet peut provenir de deux sources. Premièrement, il pourrait s'agir une autocensure des locuteurs autochtones qui obéissent aux tabous et, deuxièmement, il peut s'agir des interventions des modérateurs sur Internent, plus stricts dans certains pays que dans d'autres, contre les locuteurs qui n'obéissent pas à ces tabous, ce qui peut parler en faveur d'une certaine richesse des tabous argotiques liés à la sexualité dans ces mêmes cultures.

Dans les milieux patriarcaux, le discours des femmes par rapport à celui des hommes est toujours beaucoup plus surveillé et il est plus marqué si elles utilisent des mots obscènes, dans certains milieux mixtes où elles sont vite stigmatisées comme vulgaires et légères. Dans l'introduction du Dictionnaire érotique de Pierre Guiraud (Guiraud, 1978a, III), Alain Rey remarque : « Car le désir, le mépris et la peur des femmes ne font que transcrire dans la rhétorique des fantasmes d'une société dominée par des hommes eux-mêmes affolés par la morale judéo-chrétienne du péché ».

Puisque le vocabulaire de l'amour est riche, nous avons limité la méthodologie de ce travail à l'étude du sémantisme des mots désignant l'amour ainsi que ses manifestations sexuelles en français et dans différentes langues : bosnien, arabe, japonais et chinois et quelque peu en russe, et espagnol étant donné la longueur autorisée de l'article.

\section{Le sémantisme du mot amour en français}

Les deux mots amour et sexe sont polysémiques mais le terme amour a plus de significations. Aujourd'hui le mot amour est masculin, mais au pluriel, il est souvent employé au féminin dans la langue littéraire. Anciennement, le nom amour était féminin au singulier comme au pluriel. Voici les significations principales de ce mot en français d'aujourd'hui' ${ }^{1}$ :

\footnotetext{
${ }^{1}$ Source : https://fr.wiktionary.org/wiki/amour, consulté le 20 octobre 2019.
} 
- 'Sentiment intense et agréable qui incite les êtres à s'unir'

- 'Affection profonde pour quelqu'un ou quelque chose'

- 'Plaisir', 'intérêt'

- 'Copulation', 'relation sexuelle', 'union charnelle'

- 'Ce qui est aimé'

- 'Personne aimée'

- 'Représentation du dieu Amour, sous la forme d'un cupidon'

- 'État de fermentation propre à la végétation'

- 'Désir d'accomplir le bien d'autrui'

- (au pluriel) 'Dernières gouttes d'une bouteille de vin servies à une personne aimée'

Comme nous pouvons le voir, la plupart des significations ont le sens de amour - 'émotion', ou de amour - 'sentiment'. Mais quant à nous, nous nous intéresserons principalement à la signification 'relation sexuelle'.

\section{Le sémantisme des mots désignant le sexe en français}

Le sexe peut se référer au 'genre', à 1' 'organe sexuel' ou à l' 'acte de faire l'amour'. Nancy Huston (1980: 83) fait une classification des locutions qui font référence à l'acte sexuel en français contemporain :

1) Les termes techniques ou scientifiques - coït, copulation

2) Les euphémismes - faire l'amour, coucher avec

3) Les formules imagées - s'envoyer en l'air, se faire sauter

4) Les obscénités - baiser

5) Les gros mots - foutre

Aujourd'hui le verbe foutre est utilisé très souvent dans un sens générique pour désigner le fait d'agir en général. Si l'on dit à quelqu'un : Qu'est-c'tu fous-là ?, on ne pense plus du tout à 'l'activité de faire l'amour' (sauf dans les jeux de mots) mais à 'l'action' en général en actualisant le potentiel factitif du verbe foutre qui équivaut à faire dans beaucoup de contextes. Le verbe foutre ainsi que le verbe baiser peuvent avoir un sens figuratif. Foutre devient 'faire' par excellence mais aussi : 'mettre', 'donner', 'détruire' (Colin, Mével, Leclère, 1994 : 277) et baiser très souvent signifie 'tromper' mais aussi ‘voler' et 'comprendre' (Colin, Mével, Leclère, 1994 : 31).

\section{Comparaison des mots désignant le sexe dans les argots de différentes langues - méthodologie et corpus}

En voulant explorer les mots argotiques liés à la sexualité dans différentes langues du monde, nous avons fait une recherche sur Internet. Voulant explorer la langue parlée et vivante nous avons consulté au départ une vingtaine de sites 
d'Internet consacrés à ce sujet dont nous n'avons choisi que cinq représentatifs par leurs exemples. Ce qui nous intéressait aussi, c'était l'étymologie des métaphores utilisées. Ainsi nous avons constitué un petit corpus où l'on peut constater que le rapport à la réalité dont il est question ici n'est pas toujours le même selon les cultures mais peut parfois toucher aux mêmes interdits.

Notamment, même si la sexualité fait partie des activités quotidiennes dans toutes les sociétés, elle reste, dans la plupart d'entre elles un tabou. Certains peuples qui vivent encore dans des tribus considérées comme 'non civilisées' ont souvent un rapport plus naturel et moins complexé que nous qui sommes nés dans des sociétés dites 'civilisées'.

Dans les sociétés occidentales, connues pour leur démocratie et la liberté de la parole, une sexualité trop affichée frôle la provocation. Elle y est née aussi de la révolte contre les préceptes religieux et c'est pourquoi elle est accompagnée par la pudeur et la culpabilité touchant justement des tabous de ces mêmes préceptes religieux ( $c f$. Sokolija, $2014: 320$ ).

Que dire alors des pays et des cultures où le corps de la femme est totalement caché et couvert, sans parler même des parties du corps qui évoquent la sexualité, et où l'apparition-même de la femme fait penser au sexe et au péché. Il en va de soi que l'argot et les mots désignant la sexualité y seront niés et censurés et ces interdits sont si forts que l'on ne peut même pas trouver les mots de ce champ lexical sur Internet ou du moins on en trouve que très rarement. La sexualité dans le cas des argots des dialectes arabes, par exemple, devient un tabou culturel parce que la censure agit au niveau de toute une société aussi par autocensure et probablement par l'intermédiaire des modérateurs sur Internet de sorte qu'on trouve peu d'exemples par comparaison au japonais et surtout au chinois dans ce corpus.

Nous n'avons choisi que quelques exemples d'expressions en arabe égyptien, en japonais et en chinois. Nous avons fait exprès de nous éloigner un peu d'une approche européocentriste tout en faisant quelques comparaisons avec l'argot français, bosnien, espagnol, russe et avec la langue aborigène du Queensland. Nous allons faire une analyse de ces expressions.

\subsection{L'arabe égyptien}

Nous n'avons pas pu trouver beaucoup de mots argotiques dans d'autres dialectes arabes. Ceci est probablement étroitement lié à une forte tabouisation du sexe dans ces cultures aujourd'hui, ce qui fait que ces expressions sont fortement autocensurées par les locuteurs natifs même si des mêmes expressions sont probablement très présentes dans la langue parlée. Nous avons choisi deux expressions qui viennent de l'arabe égyptien ${ }^{2}$ :

${ }^{2}$ https://arabic.desert-sky.net/coll_insult.html, consulté le 20 octobre 2019. 
نالك - ينيك (naak - yiniik) arg. 'baiser'

- كس أمك. (kuss ummak) arg. 'chatte de ta mère' : arg. 'va te faire foutre !'

Le premier exemple est le verbe qui est entré dans la langue française avec le calque nique ta mère! par l'intermédiaire des dialectes des langues arabes magrébines ${ }^{3}$. Cette expression fait allusion au tabou des rapports sexuels avec la mère, donc le viol ou l'inceste (difficile d'être sûr qu'il s'agisse d'une ellipse du sujet ou d'un impératif) et touche à la mère, visiblement la personne « la plus sacrée » dans cette culture.

La deuxième expression en arabe égyptien, un juron, associe encore l'interlocuteur au sexe de sa mère. Un peu dans le même sens, les deux jurons suprêmes en bosnien sont jebem ti mater! (Saračević, 2003 : 125) et idi u pičku materinu! (Saračević, 2003 : 200). Le premier veut littéralement dire 'je baise ta mère' et le deuxième 'va dans la chatte de ta mère!'. Dans les deux cas, on touche au tabou suprême de la mère - génitrice et centre-même de la cellule familiale. Notamment, le premier juron est beaucoup plus insultant pour les hommes que pour les femmes (ce qu'on peut souvent détecter de par les réactions parfois violentes de ceux-ci) car il touche au lien sacré du fils à sa mère (selon les interprétations psychanalytiques) par le biais du « complexe d'Edipe enfreint » sous-entendant le viol de la mère de l'interlocuteur. En effet, l'objet désiré de l'homme, sa mère, est verbalement violé par l'interlocuteur.

N. Huston (118) cite aussi des exemples de l'anglais (motherfucker 'baiseur de ta mère'), de l'espagnole mexicain (chinga a su madre 'foutre sa mère'), du russe (ëb tvojú mat' 'baise ta mère') et de la langue aborigène du Queensland (yanta papa gobi ! ' 'tu aimes le vagin de ta mère!'). Donc, qu'il s'agisse du viol de la mère ou de l'allusion au rapport incestueux de l'interlocuteur avec sa mère, le tabou est brisé et l'effet est atteint.

\subsection{Le japonais}

Nous avons choisi quelques expressions de l'argot japonais ${ }^{5}$ liées à la sexualité : $^{\circ}$

- 赤馬

[あかいうま, akai uma] lit. : ‘cheval rouge’> ‘vagin’

3 À ce titre cf. J.-P. Goudaillier, Comment tu tchaches!, p. 206 et Colin, Mével, Leclère, 1994, p. 434-435.

${ }^{4}$ N. Huston emprunte le dernier exemple à Donald F. Thomson, (1935), « The Joking Relationships and organized Obscenity in North Queensland », American Anthropologist, n ${ }^{\circ} 37$. L'article est accessible sur Internet : https://anthrosource.onlinelibrary.wiley.com/doi/pdf/10.1525/ aa.1935.37.3.02a00100.

5 Sources : http://www.languagerealm.com/japanese/japaneseslang.php, consulté le 20 octobre 2019 et HALPERN, J. (éd.) (1999), The Kodansha Kanji Learner's Dictionary, Tokyo, Kenkyusha Limited.

${ }^{6}$ Nous n'avons pas pu vérifier ces expressions dans les dictionnaires de la langue standard japonaise et c'est pour cela d'ailleurs aussi que nous nous sommes rabattu sur Internet. 
Il s'agit d'une métaphore animalière tout comme on utilise le mot chatte ou moule pour désigner le sexe féminin en argot français. Les comparaisons sont différentes et ont peut-être à faire avec les représentations de ces animaux dans l'imaginaire populaire en question.

・味わう

[あじわう, ajiwau], lit. : 'goûter', 'savourer' > 'baiser'

- 熱いもの

[あついもの, atsui-mono], lit. : 'choses chaudes'> vulg. 'sperme'

- 穴

[あな, ana], lit. : 'trou'> 'chatte'

Dans les trois cas précédents, il s'agit de métaphores. La dernière métaphore est aussi présente en argot bosnien (rupa) (Saračević, 2003 : 232).

・あつあつになる

[あつあつになる, atsu atsu ni naru], lit : 'devenir chaud chaud'> lit. 'ils sont scotchés'> 'ils baisent'

・(あつあつ

[あつあつ, atsu-atsu], lit. 'chaud chaud'> 'baiser'

La réduplication est ici liée à la métonymie.

\subsection{Le chinois mandarin}

Les pages consacrées à l'argot chinois sur Internet sont plus nombreuses. Nous avons choisi une source relativement systématique ${ }^{7}$ de l'argot mandarin.

- 二屏 (Èr bī / ou seulement l'abréviation $2 B$ sur Internet) lit. 'double vagin' $>$ 'con', 'idiot'

- 俊屄 (shăbī / ou seulement l'abréviation $S B$ sur Internet) lit. 'con stupide' $>$ 'personne stupide'

Comme nous pouvons le voir, et en chinois et en français (con) on associe au sexe féminin la 'bêtise'. En bosnien, par contre, pička ('chatte'), en argot, peut désigner 'quelqu'un de lâche' (Saračević, 2003 : 200). Il s'agit de connotations définitivement négatives.

- Chinois simplifié : 炒饭; chinois traditionnel : 炒飯 (chăofàn), lit. 'sauté de riz'; 'riz cantonnais'> vulg. 'faire l'amour' > 'baiser'

L'acte d'amour est ici vu métaphoriquement comme une sorte de mélange, un plat.

- chinois simplifié 鸡巴; chinois traditionnel : 雞巴/鷄巴 (jībā) (sigle sur Internet : $J 8 / G 8)$, lit. 'coq'> arg. 'bite'

${ }^{7}$ Sources : https://en.wikibooks.org/wiki/Chinese_(Mandarin)/Slang et Oxford Advanced Learner's English-Chinese Dictionary 9th Edition (2018), London, Hornby, consultés le 20 octobre 2019. 
Il s'agit là d'un emploi métonymique.

- 打飞机 (dăfêijī), lit. 'manipuler un avion'> arg. 'branler (un homme)'

C'est une expression métaphorique.

- 帛 ou substitué par 吊 (diăo), vulg. 'sexe masculin', 'bite' où le même caractère signifie aussi 'copuler' en cantonais

La copulation est ici identifiée au sexe masculin (deuxième signification du caractère chinois).

Tout comme en Occident, les femmes qui sont 'portées sur le sexe' sont stigmatisées alors que les termes pour les hommes qui ont le même comportement sont rares :

- 狐狸精 (húli jīng), lit. 'esprit de renarde'> une 'femme très sexy'> 'salope'

- chinois simplifié : 公共汽车; chinois traditionnel : 公共汽車 (gōng gòng qì chē), lit. 'bus public'> 'la femme qui couche avec tout le monde', 'poufiasse'

Ceci est à comparer avec les expressions anglaises : she's the town bicycle, everyone has had a ride ou alors avec l'expression française : Marie couche-toi là !, qui désignent les deux vulgairement une 'femme légère', une 'salope'.

- 同志 (Tóngzhì), lit. 'camarade' > 'homosexuel'

Ce mot a été récemment adopté à Hong Kong et à Taiwan et il est souvent utilisé en Chine. Il veut dire littéralement 'celui qui a les mêmes aspirations'. Il a été emprunté au discours politique et s'applique maintenant aussi au discours sexuel. Étant donné le tabou politique, ce glissement sémantique est quelque peu audacieux pour la population chinoise.

\section{Conclusion}

Alors que dans la langue standard on parle plus ouvertement de l'amour que de la sexualité, le rapport s'inverse en argot. C'est probablement parce que les argots dévoilent les tabous des cultures où ils sont parlés. Il existe aussi une autre hypothèse. L'amour, en tant qu'émotion, est plutôt indicible en argot, ce qui est lié à la fonction pudique de l'argot dont parlait Denise François-Geiger (1989 : 95). Ainsi, quand on dit tu me bottes ou je te kiffe, on dit plutôt 'tu me plais' et non pas 'je t'aime' - ce qui devient paradoxalement un tabou en argot. Comme si dans la poétique de l'argot il ne convient surtout pas de dire à un autre individu qu'on l'aime.

Ceci étant dit, le statut de la femme en argot reste inférieur à celui de l'homme ou comme le formule Pierre Guiraud dans l'introduction à son ouvrage :

Cela dit, en marge de cette étude dont le principal objet est la description de la pensée analogique médiatisée par le langage, je pense avoir, implicitement montré que : Premièrement - et à partir du langage - l'existence d'une image de la sexualité qui aliène et dégrade la femme, parfois jusqu'aux limites de l'absurde et de l'obscène et aux dépens d'un rapport harmonieux et vrai entre les sexes... (Guiraud, 1978b : 13). 
La sexualité et ses tabous sont présents en argot aussi dans d'autres contextes et avec d'autres fonctions. Ainsi : les gros mots font apparaître une forte fonction expressive et impressive (Jakobson, 1963 : 216) et peuvent créer de la sorte une interaction conflictuelle entre les interlocuteurs et par leur poids évocateur émotif réveiller l'agressivité ; ils peuvent être actualisés dans le discours sexuel dans une fonction référentielle ou littérale; les gros mots ont souvent une fonction cathartique de soulagement et d'évacuation des émotions. Nous avons tous été témoins de situations où, quand nous brisons un objet sans vouloir le faire, nous prononçons des expressions qualifiées de vulgaires. C'est parce qu'en les prononçant, nous brisons les tabous et évacuons la colère.

En comparant, ne serait-ce qu'un peu, les expressions argotiques qui concernent la sexualité dans les langues citées, on peut déduire que ces expressions apparaissent dans ces différentes langues même si leur efficacité n'est pas la même parce que leur degré de tabouisation et leur poids sémantique actualisé n'est pas le même selon les cultures. Néanmoins, il semble que tous ces argots extériorisent ces tabous et, par là, ils découvrent notre inconscient à travers la langue. Et du moment que l'amour reste un sujet universel, il est très probable que sa deuxième facette, la sexualité, souvent évitée dans les langues standard, restera gravée en argots.

\section{Bibliographie}

COLIN, Jean-Paul, MÉVEL, Jean-Pierre, LECLÈRE, Christian (1994), Dictionnaire de l'argot, Paris, Larousse

FRANÇOIS-GEIGER, Denise (1989), L'argoterie, Paris, Sorbonneargot

GOUDAILLIER, Jean-Pierre (2001), Comment tu tchatches! Dictionnaire du français contemporain des cités, Paris, Maisonneuve \& Larose

GUIRAUD, Pierre (1973), L'argot, Paris, PUF

GUIRAUD, Pierre (1975), Les Gros Mots, Paris, PUF

GUIRAUD, Pierre (1978a), Dictionnaire érotique, Paris, Éditions Payot \& Rivages

GUIRAUD, Pierre (1978b), Sémiologie de la sexualité, Paris, Payot

HALPERN, Jack (éd.) (1999), The Kodansha Kanji Learner's Dictionary, Tokyo, Kenkyusha Limited HUSTON, Nancy (1980), Dire et interdire, Paris, Petite bibliothèque Payot

JAKOBSON, Roman (1963), Essais de linguistique générale, Paris, Éditions de Minuit

SARAČEVIĆ, Narcis (2003), Rječnik sarajevskog žargona, Zenica, Vrijeme

SOKOLIJA, Alma (2014a), L'argot parisien et l'argot sarajevien avec les dictionnaires, Sarajevo, Faculté de Philosophie de l'Université de Sarajevo. (http://www.ff-eizdavastvo.ba/Books/ 1\%E2\%80\%99Argot_parisien_et_1\%E2\%80\%99argot_sarajevien_avec_les_dictionnaires. pdf, consulté le 20 octobre 2019)

SOKOLIJA, Alma (2014b), « Taboo expressions and stigmatization and their manifestations in language and slang », Travaux XVII, Sarajevo, Faculté de Philosophie de l'Université de Sarajevo, p. 319-328. (http://ff-eizdavastvo.ba/Books/SerialPubl/Radovi_FFUNSA_2014.pdf, consulté le 20 octobre 2019)

THOMSON, F. Donald (1935), « The Joking Relationships and organized Obscenity in North Queensland », American Anthropologist, $\mathrm{n}^{\circ}$ 37, p. 460-490

Oxford Advanced Learner's English-Chinese Dictionary 9th Edition (2018), London, Hornby 


\section{Sitographie}

http://www.languagerealm.com/japanese/japaneseslang.php, consulté le 20 octobre 2019

https://arabic.desert-sky.net/coll_insult.html, consulté le 20 octobre 2019

https://blogs.transparent.com/chinese/swear-words-in-chinese/, consulté le 20 octobre 2019

https://en.wikibooks.org/wiki/Chinese_(Mandarin)/Slang, consulté le 20 octobre 2019

https://fr.wiktionary.org/wiki/amour, consulté le 20 octobre 2019

Alma Sokolija - professeure associée au Département de langues romanes, Université de Sarajevo, Bosnie-Herzégovine ; docteure de linguistique générale (spécialité : sociolinguistique) ; auteure de deux livres et d'une vingtaine d'articles. Ses principaux domaines d'intérêt scientifique sont les argots, les registres des langues, la langue parlée et l'analyse contrastive. 